\title{
Thermo-coupled Surface Cauchy-Born Theory: An Engineering Finite Element Approach to Modeling of Nanowire Thermomechanical Response
}

\author{
Esfahania, M. Nasr ; Sonne, Mads Rostgaard; Hattel, J. Henri; Alaca, B. Erdem
}

Published in:

Mechanics of Materials

Link to article, DOI:

10.1016/j.mechmat.2015.11.011

Publication date:

2016

Document Version

Peer reviewed version

Link back to DTU Orbit

Citation (APA):

Esfahania, M. N., Sonne, M. R., Hattel, J. H., \& Alaca, B. E. (2016). Thermo-coupled Surface Cauchy-Born Theory: An Engineering Finite Element Approach to Modeling of Nanowire Thermomechanical Response. Mechanics of Materials, 94, 46-52. https://doi.org/10.1016/j.mechmat.2015.11.011

\section{General rights}

Copyright and moral rights for the publications made accessible in the public portal are retained by the authors and/or other copyright owners and it is a condition of accessing publications that users recognise and abide by the legal requirements associated with these rights.

- Users may download and print one copy of any publication from the public portal for the purpose of private study or research.

- You may not further distribute the material or use it for any profit-making activity or commercial gain

- You may freely distribute the URL identifying the publication in the public portal 


\section{Accepted Manuscript}

Thermo-coupled Surface Cauchy-Born Theory: An Engineering Finite Element Approach to Modeling of Nanowire Thermomechanical Response

M.Nasr Esfahani, M.Rostgaard Sonne, J.Henri Hattel, B.Erdem Alaca

PII:

DOI:

Reference:

To appear in:

Received date:

Revised date:

Accepted date:
S0167-6636(15)00251-3

10.1016/j.mechmat.2015.11.011

MECMAT 2515

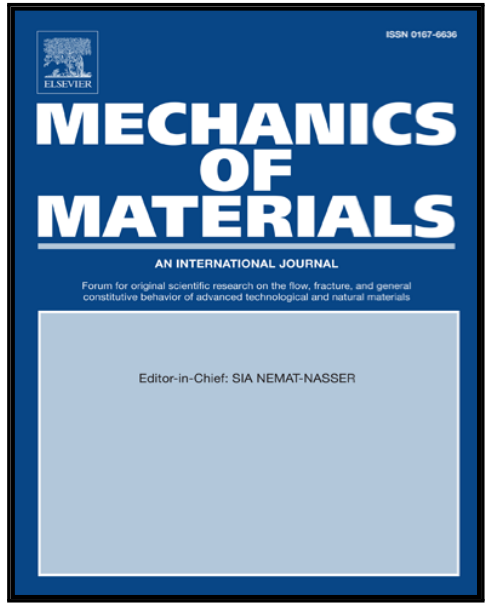

Please cite this article as: M.Nasr Esfahani, M.Rostgaard Sonne, J.Henri Hattel, B.Erdem Alaca, Thermo-coupled Surface Cauchy-Born Theory: An Engineering Finite Element Approach to Modeling of Nanowire Thermomechanical Response, Mechanics of Materials (2015), doi: 10.1016/j.mechmat.2015.11.011

This is a PDF file of an unedited manuscript that has been accepted for publication. As a service to our customers we are providing this early version of the manuscript. The manuscript will undergo copyediting, typesetting, and review of the resulting proof before it is published in its final form. Please note that during the production process errors may be discovered which could affect the content, and all legal disclaimers that apply to the journal pertain. 


\section{Highlights}

- Thermo-Coupled surface Cauchy-Born Theory for nanowire thermomechanical response is proposed.

- Surface energy included in thermomechanical modeling.

- The method is based on utilizing temperature dependent interatomic potential.

- The proposed method is verified with Molecular Dynamics simulation.

- Computational efforts are reduced without compromising physics of the problem.

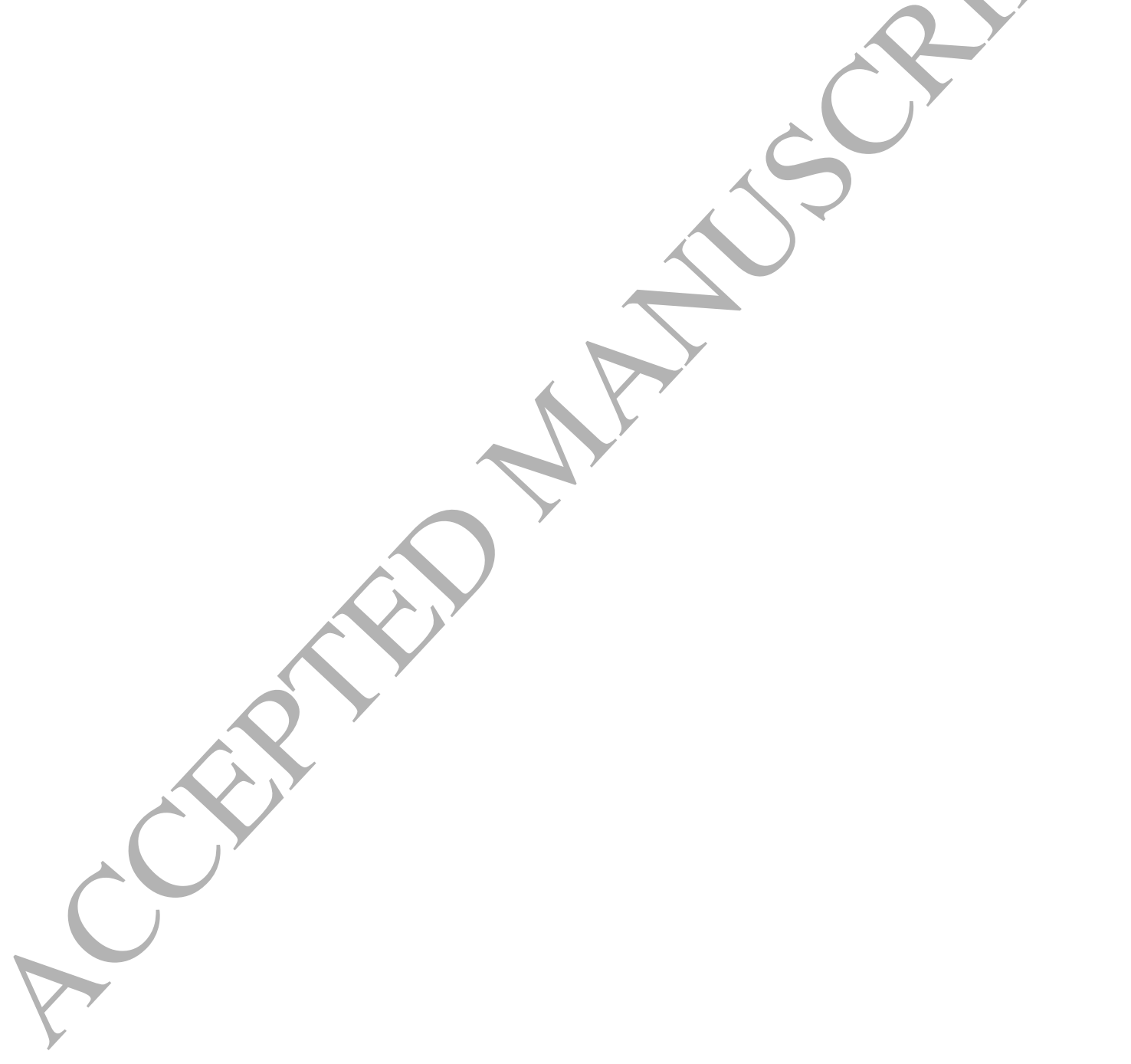




\title{
Thermo-coupled Surface Cauchy-Born Theory: An Engineering Finite Element Approach to Modeling of Nanowire Thermomechanical Response
}

\author{
M. Nasr Esfahani ${ }^{\mathrm{a}}$, M. Rostgaard Sonne ${ }^{\mathrm{b}}$, J. Henri Hattel ${ }^{\mathrm{b}}$, B. Erdem Alaca ${ }^{\mathrm{a}, \mathrm{c}, *}$ \\ ${ }^{a}$ Department of Mechanical Engineering, Koç University, Rumelifeneri Yolu, Sariyer 34450 Istanbul, Turkey \\ ${ }^{b}$ Department of Mechanical Engineering, Technical University of Denmark,building 425,2800Kgs. Lyngby, Denmark \\ ${ }^{c}$ Koç University Surface Science and Technology Center, Rumelifeneri Yolu, Sariyer 34450 Istanbul, Turkey
}

\begin{abstract}
There are remarkable studies geared towards developing thermomechanical analyses of nanowires based on quasiharmonic and Molecular Dynamics simulations. These methods exhibit limited applicability due to the associated computational cost. In this study an engineering finite-temperature model based on Surface Cauchy-Born theory is developed, where surface energy is accounted for in the prediction of the thermomechanical response, This is achieved by using a temperature-dependent interatomic potential in the standard Cauchy-Born theory with a surface energy contribution. Simultaneous calculation of thermal and mechanical stresses is achieved by eliminating the diagonalization matrix of entropy in the quasiharmonic system. This leads to a reduction in the degrees of freedom by more than $99 \%$ in comparison with equivalent Molecular Dynamics models. For the purpose of validation, results obtained on copper and nickel nanowires through the proposed method are compared with those of the more involved Molecular Dynamics simulations. This comparison verifies the significant reduction in the computational process with an acceptable accuracy. Hence, the proposed method provides a promising engineering tool without compromising the underlying physics of the problem and has potential implications in the effective modeling of the nanoscale thermomechanical behavior.
\end{abstract}

Keywords: Surface Cauchy-Bron; Temperature; Nanowire; Finite élement; Surface Energy

\section{Introduction}

Nanowires (NWs) have been under intense development in recent years due to their unique mechanical, optical and electrical properties. They are utilized in various applications including electronics (Sacchetto et al., 2009) and photonics (Yan et al., 2009). A further emerging field of their application is nanoelectromechanical systems (NEMS), where they are mainly utilized as transducers for high-resolution mass (Hanay et al., 2012) and force sensing (Sage et al., 2015). Measurements down to yoctogram (Chaste et al., 2012) and femtoNewton (Marago et al., 2008) are reported. Most of /these techniques involve the dynamic-mode operation of the NW, where changes in the resonant behavior are monitored as indicators of external mechanical effects (Gil-Santos et al., 2010; Sadek et al., 2010; Yang et al., 2006). Hence, NWs serve as ultra-small mechanical components, where size reduction proves to be extra advantageous in sensing applications. The main advantages are associated with i) their high frequencies of operation leading to high sensitivities and high resolution, ii) their ability of deterministic integration with higherorder structures such as electrodes, and iii) the ease of their use as transducers, especially through piezoresistivity in Si NWs (Erdem Alaca, 2009; He and Yang, 2006;

\footnotetext{
*Corresponding author

Email address: ealaca@ku.edu.tr (B. Erdem Alaca)
}

Sansa et al., 2014; Walther et al., 2012), and stretchable conductors with metal NWs (Xu and Zhu, 2012; Yao and Zhu, 2015).

It is well known that mechanical properties exhibit dramatic size dependence. For example, Cao and Chen (2008) reported a significant size dependence of the modulus of elasticity in ZnO NWs (Cao and Chen, 2008). Similarly, Cuenot et al. (2004). showed an increase in the modulus of elasticity in silver and lead NWs in smaller diameters (Cuenot et al., 2004). Further effects such as phase transformations in gold NWs (Diao et al., 2003) and pseudoelastic behavior in NWs made of shape memory alloys (Park et al., 2005) are linked to the reduction of size. As the mechanical behavior of NWs is core to the operation of the majority of NEMS applications, modeling of their mechanical properties becomes a critical task. It proves to be a challenging task as well, as size reduction leads to the dominance of quantum effects due to the confinement by free surfaces and interfaces (Wang and Herron, 1991). A key feature of a free surface or an interface is the difference in their atomic arrangement due to the lack of bonding neighbors. This leads to a different set of elastic properties of surfaces from that of the bulk, where fully coordinated atomic arrangements and balanced forces exist.

A wide range of analytical models for the size-dependent elastic properties at the nanoscale has recently been devel- 
oped. These models are usually based on enhanced classical continuum mechanics that use a bulk/surface decomposition to study surface effects with varying nanostructure dimensions. For example, Wang (Wang and Feng, 2009) and He (He and Lilley, 2008) presented the surface effect on bending and resonance frequency of NWs by utilizing a surface layer of finite thickness and the Young-Laplace equation (Tadmor et al., 1996b). On the contrary, an extensive range of multiscale modeling studies investigates problems with continuum methods with an atomic-scale resolution. These techniques can generally be referred to as atomistic-based continuum analyses or quasicontinuum methods. In this approach a general continuum finite element formulation is utilized to characterize the mechanical response of a given system. A finite element analysis provides a continuum system with adaptive model refinement to save fully atomistic details and supplies a multi-scale analysis capability by refining the mesh size near lattice defects and highly energetic regions such as stacking faults and dislocations (Tadmor et al., 1996b) and elastic and plastic deformations (Tadmor et al., 1996a) in solids. The main difference of the atomistic-based continuum analyses from classical finite element methods is the derivation of the constitutive response from an atomistic standpoint rather than empirical rules and phenomenological models (Tadmor et al., 1996b).

Recently, Park et al. (2006) proposed a multiscale Surface Cauchy-Born (SCB) method to capture atomic-scale surface stress effects based on decomposing the potential energy into bulk and surface components, where bulk and surface stresses constitute distinct terms in the variational equation. This decomposition is the basis of capturing surface energy as the size of the continuum body decreases and the surface energy becomes dominant(Park et al., 2006). This method was successfully utilized to demonstrate size dependence in bending behavior (Yun and Park, 2009) and resonance frequency of metallic (Park and Klein, 2008) and Si (Park, 2008) NWs due to the free surface energy. Although the aforementioned atomistic-based continuum analyses can describe phenomena such as sizedependence, surface energy and crystal non-uniformity, their use is confined to cryogenic systems. As classical interatomic potentials such as Lennard-Jones (LJ), Embedded Atom Method (EAM) and Tersoff are defined in static atomistic systems at zero absolute temperature, modeling of the thermomechanical response in nanostructures with atomistic resolution constitutes a novel challenge in engineering applications.

Only recently the temperature-dependent interatomic potential was developed as an efficient molecular calculation technique to model high-temperature behavior (Subramaniyan and Sun, 2008). For example, in Molecular Dynamics (MD) simulations, the effect of temperature is accounted for by thermal vibrations of each atom, where the system is permitted to progress dynamically by integrating Newtons equations of motion for each atom over time for the atomic system. Dupuy et al. (2005) proposed a finite-temperature method by a combination of statistical mechanics and finite element calculations (Dupuy et al., 2005). Xiao and Yang (2006), Liu and Li (2007), Jiang et al. (2005) and Tang et al. (2006) developed a multiscale Helmholtz free energy method based on the CauchyBorn approximation. In this method, the effect of the finite temperature is accounted for by the local harmonic approximation, which relates the entropy to the vibration frequencies of the system(Jiang et al., 2005; Liu and Li, 2007; Tang et al., 2006; Xiao and Yang, 2006).

The effect of free surface on mechanical properties is one of the most sought-after modeling aspects in NEMS. As NEMS designs and applications are under constant evolution, engineering approaches with straightforward, computationally less demanding numerical techniques relevant to operation conditions such as finite temperatures are highly needed. In this study, we present the thermo-coupled surface Cauchy-Born (TSCB) model, a continuum-based theory with multiscale coupling for the thermomechanical analysis of NWs at finite temperatures where surface stress has a significant contribution. Based on the developments of the MD and the Helmholz free energy methods to capture surface effects at finite temperatures, the present work proposes to implement temperature in the definition of the interatomic potential as opposed to using temperature as a kinetic component in entropy calculations from quasiharmonic approximations. This is achieved by utilizing Engineering Molecular Mechanics (EMM) proposed by Subramaniyan and Sun (2008) as an alternative molecular simulation tool (Subramaniyan and Sun, 2008) within the SCB method. Compared to MD and quasiharmonic models the proposed method requires less computational power and provides a bridge between fundamental scientific modeling efforts and practical engineering applications without compromising accuracy.

In the remainder of this work the Surface Cauchy-Born hypothesis is introduced first, followed by the description of the combination of molecular mechanics with finite element calculations. The results of the finite element simulations are compared with traditional MD calculations for validation. The work is concluded by analyzing the accuracy of the proposed method for the prediction of the modulus of elasticity as an essential parameter in mechanical response modeling. Finally, the opposing effects of surface and thermal energies on the mechanical response are demonstrated.

\section{Methodology}

The quasicontinuum method has been developed to investigate the mechanical response of a continuum body with atomic scale interactions. In this approach, a classical continuum finite element formulation is employed to characterize the mechanical response of a given system, where the constitutive response of the system is determined by interatomic calculations. The basic idea is that 
every point in a continuum corresponds to a very large region on the atomic scale. Thus, the constitutive response at that point may be obtained by deforming the underlying crystal structure by the local strain to obtain the local state of stress. This constitutive response is derived by utilizing ordinary interatomic interactions such as LJ and EAM for metals and Tersoff potential for covalent systems.

Since the quasicontinuum method does not account for the surface energy contribution at the nanoscale, the SCB model was developed by Park et al. (2006) to capture surface effects within the framework of the atomic-based continuum analysis. In this method, the energy of the system is divided into bulk and surface components. The interatomic potential can be turned into the Bulk Cauchy-Born approximation by two steps: converting the potential energy into a strain energy density through normalization by the atomic volume, $\Omega_{0}$, and then deforming the neighbors around each atom homogeneously via continuum mechanics quantities such as the deformation gradient $\mathrm{F}$, or right stretch tensor $C=F^{T} \mathrm{~F}$ (Park et al., 2006). As the result, the strain energy density at finite temperatures, $\varphi(C, T)$, can be expressed as a function of the temperature-related interatomic pair potential, UT, as

$\varphi(C, T)=\frac{1}{2 \Omega_{0}} \sum_{m=1}^{n b} U_{T}\left(r^{m}(C), T\right)$

where $n b$ is the total number of bonds to a representative bulk atoms, $\Omega_{0}$ is the atomic volume in the reference configuration with undeformed bond vector $R^{m}$, and $r^{m}$ is the deformed bond length at atom $m$ and its neighbors

$r^{m}=\sqrt{R^{m} \cdot C R^{m}}$

This energy equivalence forms the basis of the traditional CB hypothesis, in which lattice defects are not considered. When the strain energy function at finite temperatures is known, the continuum stress can be derived by differentiating the material strain energy density function with respect to the right stretch tensor, where there is no need to divide the stress into mechanical and thermal portions (Yun and Park, 2008). The second Piola-Kirchoff stress at finite temperature can be written as

$$
S_{T}=2 \frac{\partial \varphi(C, T)}{\partial C}
$$

whereas the temperature-related spatial modulus $D_{T}$ is defined as

$D_{T}=4 \frac{\partial^{2} \varphi(C, T)}{\partial C_{i j} \partial C_{k l}}$

The lack of any decomposition requirement of stress and modulus into their mechanical and thermal components provides a significant advantage in comparison to the Helmholz free energy methods. All points in the bulk are considered in equilibrium with a fully coordinated atomic structure in the BCB hypothesis and all points on the surface have fewer bonding neighbors that leads to a surface stress. Therefore, the SCB was developed to include surface energy in NWs by capturing surface stress due to the uncoordinated atoms on the surface. The strain energy density of a body can be expressed in bulk and surface terms by decomposing the potential energy into bulk and surface components. The relationship between the continuum strain energy density and the total potential energy of a defect-free system at a finite temperature can thus be expressed as

$$
\sum_{m=1}^{n_{\text {atom }}} U_{T}^{m}(r, T)=\int_{\Pi_{0}} \varphi(C, T) d \Omega+\int_{\Gamma_{0}} \chi(C, T) d \Gamma
$$

where $\varphi(C, T)$ is the bulk straín energy density integrated over the volume of the body, $\Pi_{0}$, in which all atoms are fully coordinated and $\gamma(C, T)$ is the surface strain energy density, integrated in each non-bulk surface layer of the body, $\Gamma_{0}$, in which the atoms are not coordinated at high temperatures. The strain energy density on the surface at a finite temperature can be written as

$$
\gamma(C, T)=\frac{1}{2 \Gamma_{0}} \sum_{m=1}^{n b s} U_{T}\left(r^{m}(C), T\right)
$$

where $n b s$ is the number of bonds for an atom on the surface. Similar to the bulk continuum thermomechanical stress, the surface Piola-Kirchoff stresses at high temperatures can be defined as

$\widetilde{S_{T}}=2 \frac{\partial \gamma(C, T)}{\partial C}$

where the surface stress, $\widetilde{S_{T}}$, is a $3 \times 3$ tensor with normal components which allows surface relaxation as the atoms lying at the surface are not fully coordinated. This stress formulation is different from the conventional definition of a $2 \times 2$ surface stress (Shenoy, 2005) which includes only tangential terms. Similarly, the surface spatial modulus, $\widehat{D_{T}}$, can be defined as

$\widetilde{D_{T}}=4 \frac{\partial^{2} \gamma(C, T)}{\partial C_{i j} \partial C_{k l}}$

The total potential energy at finite temperature for the continuum body in which the temperature-related strain energy was decomposed into bulk and surface components under an external load $\bar{T}$ can be written as

$\Psi(u, T)=\int_{\Pi_{0}} \varphi(C, T) d \Omega+\int_{\Gamma_{0}} \gamma(C, T) d \Gamma-\int(\bar{T} . u) d \Gamma$

To obtain a suitable form for the finite element calculation, the standard discretization of the displacement field $u(X)$ can be utilized by using shape functions as

$u(X)=\sum_{I=1}^{n n} N_{I}(X) u_{I}$ 
where $N_{I}$ represent the shape or interpolation functions, $n n$ are the total number of nodes in the discretized continuum and $u_{I}$ represent the displacements of node $I$. Differentiating equation (9) with respect to the nodal displacement at the minimum potential energy gives the finite element nodal load balance as

$\frac{\partial \Psi}{\partial u_{I}}=\int_{\Pi_{0}} B^{T} S_{T} F^{T} d \Gamma+\int_{\Gamma_{0}} B^{T} \widetilde{S_{T}} F^{T} d \Gamma-\int_{\Gamma_{T}} N_{I} \bar{T} d \Gamma$

where $B^{T}=\left(\left(\partial N_{I}\right) / \partial X\right)^{T}$ is the strain displacement matrix. Eqn. (11) requires an iterative method to solve the non-linear system of equations to determine the nodal displacements that minimize the energy of the continuum body.

The strain energy density at a finite temperature in the crystal structure can be derived by using EMM, which was developed for temperature-dependent interatomic potentials to analyze static MD at high temperatures. The interatomic potential in MD simulations and the Helmholz free energy are temperature independent, i.e. temperature effects are included by allowing the system to evolve dynamically according to the Newtons equation of motion and kinetic energy in entropy calculation, respectively. In the EMM (Subramaniyan and Sun, 2008), the thermal vibration of each atom is replaced with a few parameters in the interatomic potential to capture the global averaged temperature-dependent behavior of the system. It has thus been proposed to use thermal expansion to represent the effect of temperature in materials at the atomic level. The LJ potential taking the temperature dependence into account can be expressed as

$U_{T}(r)=\epsilon_{T}\left[\left(\frac{R_{T}}{r}\right)^{12}-2\left(\frac{R_{T}}{r}\right)^{6}\right]$

where

$R_{T}=(1+\alpha T) R_{0}$

$\epsilon_{T}=-\left[U_{0}\left(R_{T}\right)+k_{B} T\right]$

in which $k_{B}$ is the Boltzmann constant, $R_{T}$ and $\epsilon_{T}$ are the LJ parameters at temperature $T$, and $U_{T}$ is the temperature-dependent interatomic potential. $R_{0}$ and $\alpha$ are interatomic distance at $0 \mathrm{~K}$ and thermal expansion coefficient, respectively.

In the present work, the temperature-dependent interatomic potential is utilized to study surface energy at finite temperatures, where the TSCB method is developed as an alternative to the MD and quasiharmonic models for crystalline solids at finite temperatures. Simultaneous derivation of mechanical and thermal stresses makes the technique easier to implement and leads to a significant increase in efficiency. For this purpose we propose utilizing the temperature-dependent interatomic potential turning the quasicontinuum method more practical for engineering applications. To evaluate the accuracy of the proposed method, a numerical study is conducted on copper and nickel NWs, where interactions in an FCC crystal are modeled using temperature-dependent LJ potentials and results are compared with those of MD simulations.

\section{Numerical Model}

All numerical examples are presented for the FCC single-crystalline $\mathrm{Cu}$ and $\mathrm{Ni} \mathrm{NWs}$ oriented along the $<100>$ direction with $\{100\}$ surfaces. The NWs have a square cross-section of $30 \times 30$ unit cells and a length of 100 unit cells. The atomic interactions are modeled using temperature-dependent LJ interatomic potentials (Subramaniyan and Sun, 2008) with a truncation after the third shell of the nearest neighbors. This approach leads to two non-bulk layers of atoms at the surfaces as illustrated in Fig.1.

All SCB calculations, utilize regular eight-node hexahedral elements. The temperature-dependent interatomic potential at different temperatures is considered with the following parameters (Subramaniyan and Sun, 2008) for copper $\left(R_{0}=2.6 \AA ; \quad \epsilon_{0}=0.167 \mathrm{eV}\right.$ and $\left.\alpha=17 \times 10^{-6} K^{-1}\right)$ and for $\operatorname{nickel}\left(R_{0}=2.258 \AA\right.$; $\epsilon_{0}=0.195 \mathrm{eV}$ and $\left.\alpha=13 \times 10^{-6} \mathrm{~K}^{-1}\right)$.

NWs are defined in the form of cantilevers, fixed at one end and free to move at the other one for surface stress relaxation (Fig.1). In the first step, no external loads are applied and all deformations obtained are due to the thermomechanical surface stresses.

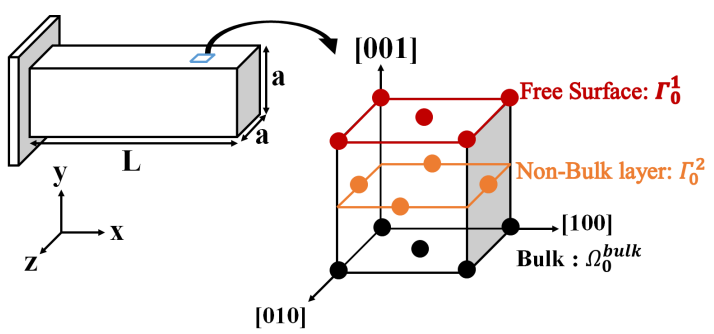

Figure 1: NW geometry considered for surface stress relaxation (Left) and Bulk and Non-bulk layers in crystal orientation (Right).

In the second step, uniaxial tension is applied along the $<100>$ direction of the NWs up to a strain of $0.2 \%$ at different temperatures. The modulus of elasticity is extracted from the resulting stress-strain curves at finite temperatures. All numerical simulations based on the proposed method are compared with fully atomistic calculations obtained by the Sandia-developed simulation code LAMMPS (Plimpton, 1995). The same LJ potentials for NWs containing 373961 atoms are used in the MD simulations. A time step of $1 \mathrm{fs}$ is used for the numerical integration. The system is equilibrated for $80 \mathrm{ps}$ in a Nose-Hoover (Nosé, 1984) canonical (NVT) ensemble and 60 ps in a microcanonical (NVE) ensemble.

This is then followed by the application of strain along the NWs under a canonical ensemble. The equivalent 
isothermal SCB model contains a regular mesh of 1323 elements and 1792 nodes, which results in a $99.5 \%$ decrease in the number of the degrees of freedom as compared to the full-blown MD simulation. The volume integral in (11) is taken using $2 \times 2 \times 2$ Gaussian quadrature, while the surface integrals are evaluated using $2 \times 2$ Gaussian quadrature. The FEM solution is obtained by utilizing a non-linear Newton solver.

\section{Results and Discussion}

Initially, the numerical calculation based on the proposed method for the copper and nickel NWs at 0K is compared with the MD calculation. Due to the surface stress, the free end of the NW contracts upon relaxation. The longitudinal displacements found by the proposed model and MD calculations at $0 \mathrm{~K}$ are shown in Fig.2. The proposed numerical method replicates the displacement profile in the longitudinal direction quite well, where a compressive strain of $-0.414 \%$ and $-0.098 \%$ are predicted, while the MD calculation predicted a contraction of $-0.431 \%$ and $-0.11 \%$ for $\mathrm{Cu}$ and Ni NWs, respectively. As predicted in previous literature (Park et al., 2006), due to the surface stress at $0 \mathrm{~K}$, the free end contracts, thus resulting in a compressive strain in the NW. This compressive strain is dramatically geometrical dependent, where long and slender NWs with higher transverse surface area endure a higher compressive strain compared to short and thick NWs (Yun and Park, 2009).
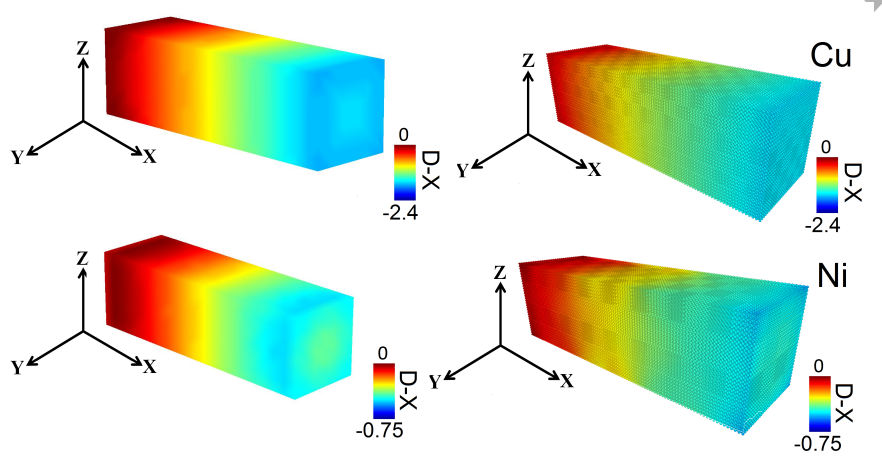

Figure 2: $\mathrm{x}$-displacement for copper and nickel NWs with $100 \times 30 \times 30$ unit cells at $0 \mathrm{~K}$ for TSCB (left) and MD (right).

Analyzing and comparing the proposed method with corresponding MD calculations include the following three aspects:

1. Axial and transverse displacement profile in the proposed method vs. MD calculation for $\mathrm{Cu} \mathrm{NWs,}$

2. Longitudinal strain of the stress-free system in the proposed method vs. MD simulation, and

3. Modulus of elasticity for the proposed model and MD calculations at different temperatures for $\mathrm{Cu}$ and $\mathrm{Ni}$ NWs.
In the first step, the TSCB calculation for a relaxed cantilever is compared with a snapshot of the MD calculations at $300 \mathrm{~K}, 400 \mathrm{~K}$ and $500 \mathrm{~K}$. The displacement profile in the x-direction for both TSCB and MD simulations are shown in Fig.3. The TSCB model replicates the displacement field in the $\mathrm{x}$-direction at different temperatures to a good degree. It shows that the proposed multiscale approach can accurately capture the effect of thermal stress and surface stress in nanostructures at the same time.

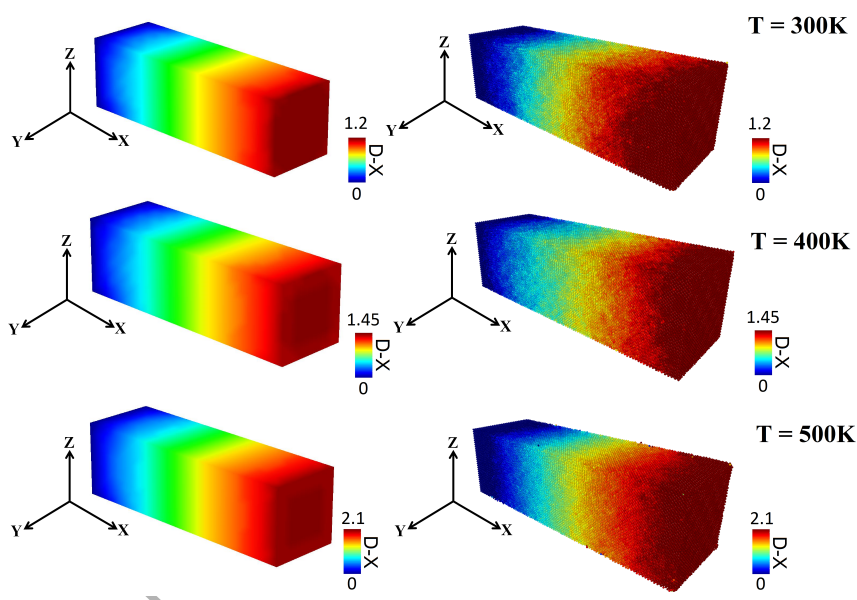

Figure 3: $\mathrm{x}$-displacement for $31.6 \mathrm{~nm} \times 10.8 \mathrm{~nm} \times 10.8 \mathrm{~nm}$ copper nanowire for TSCB (left) and the MD method (right) at $300 \mathrm{~K}, 400 \mathrm{~K}$ and $500 \mathrm{~K}$.

The displacement in the transverse direction for the MD simulation is then calculated. The results are compared once again with those provided by the proposed model. The overall snapshot of the y-displacements at $500 \mathrm{~K}$ for the TSCB and MD studies are shown in Fig.4. A good correspondence is observed as a result.

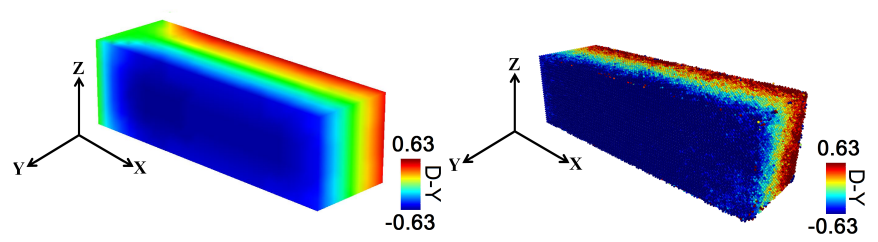

Figure 4: y-displacement field for $31.6 \mathrm{~nm} \times 10.8 \mathrm{~nm} \times 10.8 \mathrm{~nm}$ copper nanowire for TSCB (left) and the MD (right) at $500 \mathrm{~K}$.

In the second step, the longitudinal strain for relaxed copper and nickel NWs at finite temperatures for both TSCB and MD simulations are calculated to further evaluate the accuracy of the proposed method in the estimation of the surface and thermal energies. This is achieved by measuring the displacement at the free end (Fig.5). Results indicate a good agreement between the TSCB and MD methods. We note that the effects of edges and corners are ignored, where the small nanostructures with effective edges and corners (Zhao and Yakobson, 2003) can be studied directly through the atomistic calculations.

The modulus of elasticity is a key parameter when 


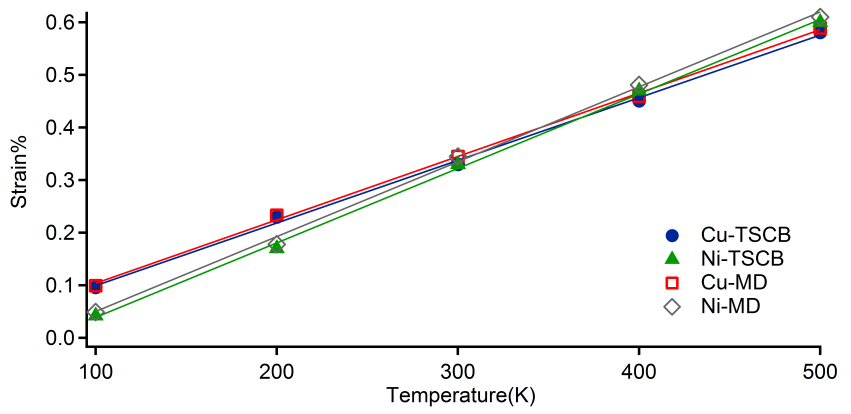

Figure 5: Uniaxial strain due to the interaction between surface and thermal stresses for $100 \times 30 \times 30$ unit cells copper and nickel NWs at different temperatures for TSCB and MD calculations.

studying the mechanical response of materials under static and dynamic loads. In the third step of validation, this fundamental parameter as predicted by the proposed method is compared with full, atomistic simulation results (Fig.6). Once again, the results demonstrate a good agreement between the TSCB and MD methods in predicting the linear elastic response of NWs at temperatures below $500 \mathrm{~K}$. The capability of the TSCB method over $500 \mathrm{~K}$ can be considered in a future study in the absence of any phase transformations, dislocation nucleation, and other crystal defects. Otherwise, non-local quasicontinuum methods should be used.

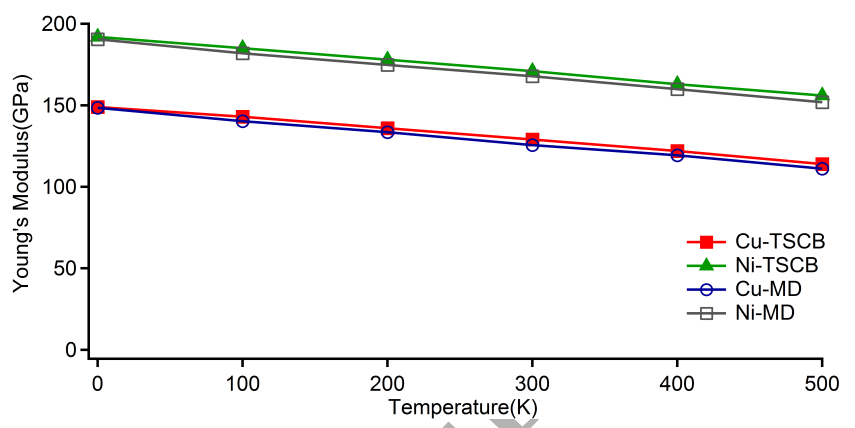

Figure 6: Youngs modulus at different temperatures predicted by TSCB and MD calculations.

Finally, the capability of the proposed method for bulk structures without surface stress is validated by experimental results in the temperature range from $4 \mathrm{~K}$ to $300 \mathrm{~K}$. Measured elastic properties of a copper single crystal in the $<100>$ direction based on the ultrasonic pulse technique (Overton Jr and Gaffney, 1955) was compared with the proposed method for the bulk strain energy, where the surface term in equation (11) was eliminated (Fig.7). The predicted elastic properties based on the proposed method have less than $8 \%$ error in comparison with experimental measurements verifying the proficiency of the proposed method for thermomechanical analysis of bulk FCC crystal structures as well.

Implementing temperature-dependent interatomic potentials in the SCB hypothesis was proposed by this work

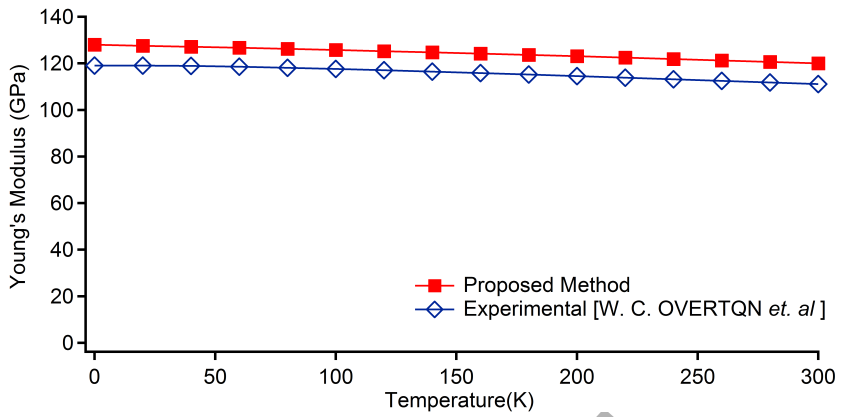

Figure 7: Youngs modulus at different temperatures predicted by the proposed method and experimentally.

to study thermomechanical behavior of NWs. Thermomechanical calculation on relaxed and strained NWs proved the accuracy of the proposed model. Predicted elastic constants for NWs with surface/energy contribution exhibit a higher stiffness compared to those reported by experimental studies for bulk crystal (Overton Jr and Gaffney, 1955).This shows the effect of surface atoms on the mechanical response of NWs. We note that predicted elastic constants and uniaxial strains are in the $\langle 100\rangle$ crystal orientation. Thermomechanical properties along other crystal orientations can be considered in future studies.

The overall investigation is based on the two-body Lennard-Jones interatomic potential in metals with linear embedded energy (Foiles et al., 1986). The temperaturedependence in interatomic potentials is included in the finite element calculations. For metals with non-linear embedded functions such as gold (Foiles et al., 1986), temperature-dependent embedded atom method (EAM) can be used instead (Subramaniyan and Sun, 2010). The proposed method is applicable for defect-free single crystals. To include any imperfection in the crystal structure, non-local quasicontinuum model (Tadmor et al., 1996b) can be utilized.

\section{Conclusion}

We present the Thermo-coupled Surface Cauchy-Born method (TSCB), a multiscale coupling continuum-based theory at finite temperatures for the thermomechanical analysis of NWs where surface stress has a significant contribution. The method is based on utilizing temperature-dependent strain energy density regarding EMM temperature-dependent interatomic potentials. This method provides a good accuracy and it is shown to be computationally much less demanding in comparison with available methods for the thermomechanical analysis with surface effects such as MD and quasiharmonic methods. The major outcome of this study is the possibility of utilizing temperature-dependent interatomic potentials to derive strain energy density for modeling surface effects. Implications of this work can be summed up as follows: 
- The possibility of coupling a temperature-dependent interatomic potential with the Cauchy-Born hypothesis for thermomechanical investigations in NWs and similar nanomechanical components.

- Simplifications in calculations and dramatic reduction in computational efforts without a significant loss of accuracy. This provides a promising engineering tool without compromising the underlying physics of the problem.

- The dominance of thermal stress over mechanical stress with increasing temperature leading to a transition in the NW strain profile from contraction to expansion.

Overall the performance of the TSCB technique in terms of simulation accuracy and computational demand is evaluated very positively. This initial study can further evolve to the derivation of momentum and heat equations in 3-dimensional nanostructures where surface stress effects and size-dependency are taken into the account.

\section{Acknowledgments}

The authors gratefully acknowledge the support by Tubitak under grant no. 112E058 and Erasmus program.

\section{References}

Cao, G., Chen, X., 2008. Size dependence and orientation dependence of elastic properties of zno nanofilms. International journal of solids and structures 45 (6), 1730-1753.

Chaste, J., Eichler, A., Moser, J., Ceballos, G., Rurali, R., Bachtold A., 2012. A nanomechanical mass sensor with yoctogram resolution. Nature nanotechnology 7 (5), 301-304.

Cuenot, S., Frétigny, C., Demoustier-Champagne, S., Nysten, B. 2004. Surface tension effect on the mechanical properties of nanomaterials measured by atomic force microscopy. Physical Review B 69 (16), 165410.

Diao, J., Gall, K., Dunn, M. L., 2003. Surface-stress-induced phase transformation in metal nanowires. Nature materials 2 (10), 656660.

Dupuy, L. M., Tadmor, E. B., Miller, R. E., Phillips, R., 2005. Finitetemperature quasicontinuum: Molecular dynamics without all the atoms. Physical Review Letters 95 (6), 060202.

Erdem Alaca, B., 2009. Integration of one-dimensional nanostructures with microsystems: an overview. International Materials Reviews 54 (5), 245-282.

Foiles, S., Baskes, M., Daw, M., 1986. Embedded-atom-method functions for the fcc metals $\mathrm{cu}, \mathrm{ag}$, au, ni, pd, pt, and their alloys. Physical Review B 33 (12), 7983.

Gil-Santos, E., Ramos, D., Martínez, J., Fernández-Regúlez, M., García, R., San Paulo, Á., Calleja, M., Tamayo, J., 2010. Nanomechanical mass sensing and stiffness spectrometry based on twodimensional vibrations of resonant nanowires. Nature nanotechnology 5 (9), 641-645.

Hanay, M., Kelber, S., Naik, A., Chi, D., Hentz, S., Bullard, E., Colinet, E., Duraffourg, L., Roukes, M., 2012. Single-protein nanomechanical mass spectrometry in real time. Nature nanotechnology 7 (9), 602-608.

He, J., Lilley, C. M., 2008. Surface effect on the elastic behavior of static bending nanowires. Nano Letters 8 (7), 1798-1802.

He, R., Yang, P., 2006. Giant piezoresistance effect in silicon nanowires. Nature nanotechnology 1 (1), 42-46.

Jiang, H., Huang, Y., Hwang, K., 2005. A finite-temperature continuum theory based on interatomic potentials. Journal of engineering materials and technology 127 (4), 408-416.
Liu, X., Li, S., 2007. Nonequilibrium multiscale computational model. The Journal of chemical physics 126 (12), 124105.

Marago, O., Jones, P., Bonaccorso, F., Scardaci, V., Gucciardi, P., Rozhin, A., Ferrari, A., 2008. Femtonewton force sensing with optically trapped nanotubes. Nano letters 8 (10), 3211-3216.

Nosé, S., 1984. A molecular dynamics method for simulations in the canonical ensemble. Molecular physics 52 (2), 255-268.

Overton Jr, W., Gaffney, J., 1955. Temperature variation of the elastic constants of cubic elements. i. copper. Physical Review 98 (4), 969.

Park, H. S., 2008. Surface stress effects on the resonant properties of silicon nanowires. Journal of Applied Physics 103 (12), 123504.

Park, H. S., Gall, K., Zimmerman, J. A., 2005. Shape memory and pseudoelasticity in metal nanowires. Physical Review Letters 95 (25), 255504.

Park, H. S., Klein, P. A., 2008. Surface stress effects on the resonant properties of metal nanowires: the importance of finite deformation kinematics and the impact of the residual surface stress. Journal of the Mechanics and Physics of Solids 56 (11), 3144-3166.

Park, H. S., Klein, P. A., Wagner, G. J., 2006. A surface cauchy-born model for nanoscale materials. International Journal for Numerical Methods in Engineering 68 (10), 1072-1095.

Plimpton, S., 1995. Fast parallel algorithms for short-range molecular dynamics. Journal of computational physics 117 (1), 1-19.

Sacchetto, D., Ben-Jamaa, M. H., De Micheli, G., Leblebici, Y., 2009. Fabrication and characterization of vertically stacked gateall-around si nanowire fet arrays. In: Solid State Device Research Conference, 2009. ESSDERC'09. Proceedings of the European. IEEE, pp. 245-248.

Sadek, A. S., Karabalin, R. B., Du, J., Roukes, M. L., Koch, C., Masmanidis, S. C., 2010. Wiring nanoscale biosensors with piezoelectric nanomechanical resonators. Nano letters 10 (5), 1769-1773.

Sage, E., Brenac, A., Alava, T., Morel, R., Dupré, C., Hanay, M. S., Roukes, M. L., Duraffourg, L., Masselon, C., Hentz, S., 2015. Neutral particle mass spectrometry with nanomechanical systems. Nature communications 6.

Sánsa, M., Fernández-Regúlez, M., Llobet, J., San Paulo, Á., PérezMurano, F., 2014. High-sensitivity linear piezoresistive transduction for nanomechanical beam resonators. Nature communications 5 .

Shenoy, V. B., 2005. Atomistic calculations of elastic properties of metallic fcc crystal surfaces. Physical Review B 71 (9), 094104.

Subramaniyan, A. K., Sun, C., 2008. Engineering molecular mechanics: an efficient static high temperature molecular simulation technique. Nanotechnology 19 (28), 285706.

Subramaniyan, A. K., Sun, C., 2010. Temperature dependent effective embedded atom method potential for steady state high temperature applications. Journal of Computational and Theoretical Nanoscience 7 (1), 176-181.

Tadmor, E., Phillips, R., Ortiz, M., 1996a. Mixed atomistic and continuum models of deformation in solids. Langmuir 12 (19), 4529-4534.

Tadmor, E. B., Ortiz, M., Phillips, R., 1996b. Quasicontinuum analysis of defects in solids. Philosophical magazine A 73 (6), 15291563.

Tang, Z., Zhao, H., Li, G., Aluru, N., 2006. Finite-temperature quasicontinuum method for multiscale analysis of silicon nanostructures. Physical Review B 74 (6), 064110.

Walther, A., Savoye, M., Jourdan, G., Renaux, P., Souchon, F., Robert, P., Blanc, C. L., Delorme, N., Gigan, O., Lejuste, C., 2012. 3-axis gyroscope with si nanogage piezo-resistive detection. In: Micro Electro Mechanical Systems (MEMS), 2012 IEEE 25th International Conference on. IEEE, pp. 480-483.

Wang, G.-F., Feng, X.-Q., 2009. Surface effects on buckling of nanowires under uniaxial compression. Applied physics letters 94 (14), 141913.

Wang, Y., Herron, N., 1991. Nanometer-sized semiconductor clusters: materials synthesis, quantum size effects, and photophysical properties. The Journal of Physical Chemistry 95 (2), 525-532.

Xiao, S., Yang, W., 2006. Temperature-related cauchy-born rule for multiscale modeling of crystalline solids. Computational Materials 
Science 37 (3), 374-379.

Xu, F., Zhu, Y., 2012. Highly conductive and stretchable silver nanowire conductors. Advanced Materials 24 (37), 5117-5122.

Yan, R., Gargas, D., Yang, P., 2009. Nanowire photonics. Nature Photonics 3 (10), 569-576.

Yang, Y., Callegari, C., Feng, X., Ekinci, K., Roukes, M., 2006. Zeptogram-scale nanomechanical mass sensing. Nano letters 6 (4), $583-586$.

Yao, S., Zhu, Y., 2015. Nanomaterial-enabled stretchable conductors: Strategies, materials and devices. Advanced Materials 27 (9), 1480-1511.

Yun, G., Park, H. S., 2008. A multiscale, finite deformation formulation for surface stress effects on the coupled thermomechanical behavior of nanomaterials. Computer Methods in Applied Mechanics and Engineering 197 (41), 3337-3350.

Yun, G., Park, H. S., 2009. Surface stress effects on the bending properties of fcc metal nanowires. Physical Review B 79 (19), 195421.

Zhao, Y., Yakobson, B. I., 2003. What is the ground-state structure of the thinnest si nanowires? Physical review letters 91 (3), 035501.

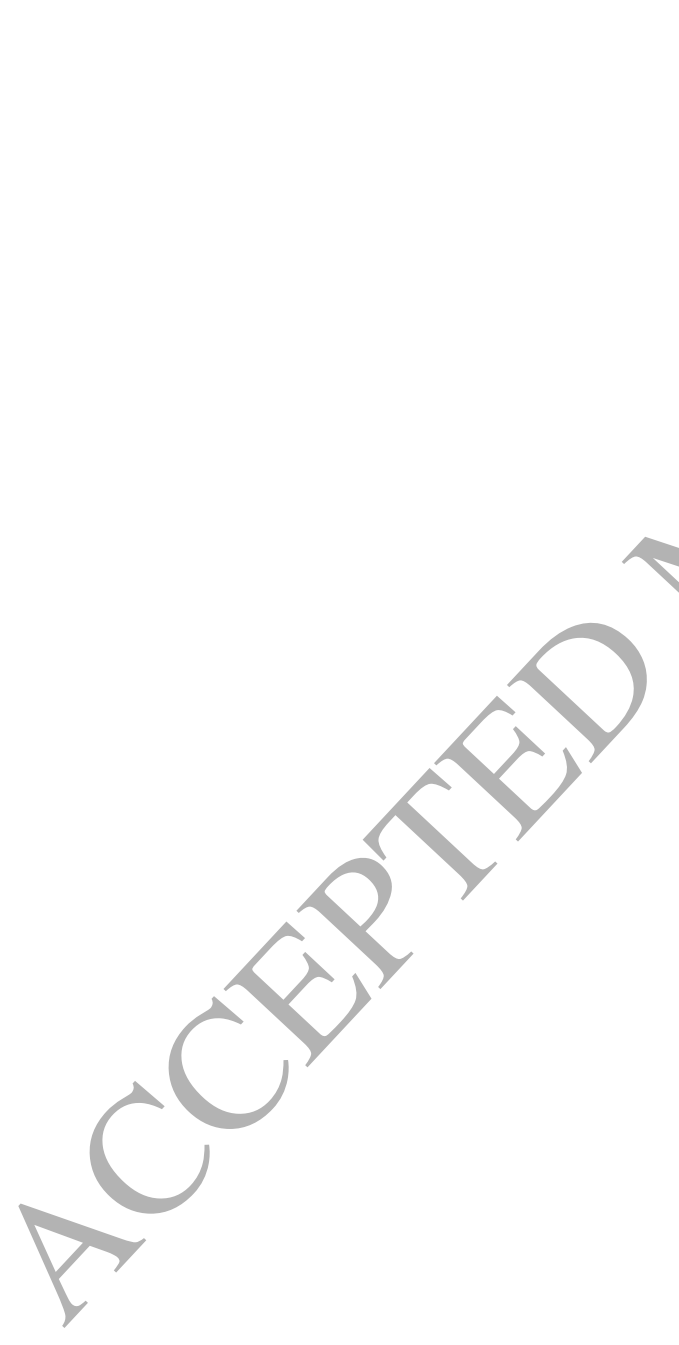

